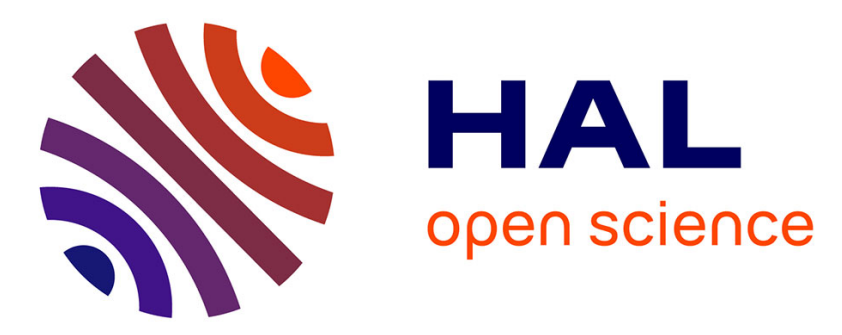

\title{
Les approches plurielles: vecteurs du développement de la compétence métalinguistique des collégiens
}

\author{
Rebecca Dahm
}

\section{To cite this version:}

Rebecca Dahm. Les approches plurielles: vecteurs du développement de la compétence métalinguistique des collégiens. Recherche et Pratiques Pédagogiques en Langues de Spécialité: Cahiers de l'APLIUT, 2014, Pratiques émergentes et recherches en didactique de l'anglais: jalons, interrogations et perspectives, XXXIII (2), pp.72-99. 10.4000/apliut.4404 . hal-01426944

\section{HAL Id: hal-01426944 \\ https://hal-univ-tlse2.archives-ouvertes.fr/hal-01426944}

Submitted on 5 Jan 2017

HAL is a multi-disciplinary open access archive for the deposit and dissemination of scientific research documents, whether they are published or not. The documents may come from teaching and research institutions in France or abroad, or from public or private research centers.
L'archive ouverte pluridisciplinaire HAL, est destinée au dépôt et à la diffusion de documents scientifiques de niveau recherche, publiés ou non, émanant des établissements d'enseignement et de recherche français ou étrangers, des laboratoires publics ou privés. 


\title{
Les approches plurielles : vecteurs du développement de la compétence méta- linguistique des collégiens
}

\section{Résumé}

Cet article cherche à décrire les processus cognitifs mis en jeu par la mise en œuvre d'une approche plurielle fondée sur des langues inconnues (APLI) au sein du cours d'anglais en classe de cinquième. Il s'agit, en effet, d'examiner les liens cognitifs et linguistiques que les élèves établissent entre les langues inconnues observées (néerlandais, italien puis finnois) dans le cadre d'activités métasémantiques inscrites dans l'APLI et les langues de leur répertoire linguistique (L1-français et L2-anglais). Les APLI aident l'élève à développer sa compétence métalinguistique puisque la résolution du problème d'accès au sens nécessite la conscientisation des savoirs et savoir-faire acquis de manière antérieure à la séance d'APLI. En outre, il apparait que les stratégies de comparaison et de traduction naturellement mises en œuvre par les élèves s'appuient avant tout sur des connaissances déclaratives alors que la déduction et l'inférence nécessitent à la fois des connaissances déclaratives et procédurales.

Pluralistic approaches: a means to develop the metalinguistic competence of lower-secondary students

\begin{abstract}
This article seeks to describe the cognitive processes involved in the implementation of a pluralistic approach based on unknown languages (PAUL) during a year-7 English class. The cognitive and linguistic links that students set up between the unknown languages (Dutch, Italian and Finnish) they consecutively observe and the languages of their multilingual repertoire (L1-French and L2-English) are being examined. The PAUL sessions help students to develop their metalinguistic competence since the access to meaning problem-solving requires the awareness of the knowledge and skills they have acquired prior to the PAUL session. It also appears that the strategies of comparison and translation naturally implemented by the students rely primarily on declarative knowledge whereas the deduction and inferencing strategies require both declarative and procedural knowledge.
\end{abstract}

\section{Mots-clés}

activités métasémantiques, compétence métalinguistique, compétence plurilingue, connaissances déclaratives, connaissances procédurales, conscientisation, processus d'apprentissage, stratégies d'apprentissage

\section{Keywords}

metasemantic activities, metalinguistic competence, multilingual competence, declarative knowledge, procedural knowledge, language awareness, learning processes, learning strategies 


\section{Les approches plurielles : vecteurs du développement de la compétence métalinguistique des collégiens}

\section{Introduction}

Intégrer les approches plurielles (Candelier, 2003) dans l'enseignement de l'anglais, c'est souhaiter contribuer au développement de la compétence plurilingue des élèves. Or, l'un des éléments constitutifs de la didactique du plurilinguisme est la réflexion métalinguistique qui développe la capacité de décentration de l'élève (Beacco et al., 2010 : 39). Il s'agit donc de susciter, chez celui-ci, une conscientisation de son répertoire pluriel afin qu'il soit en mesure de mobiliser ses ressources d'origines diverses par des stratégies efficaces favorisant l'appropriation (Castellotti \& Moore, 2011).

Or, «mettre en œuvre une compétence revient à savoir mobiliser efficacement les savoirs, savoir-faire et savoir-être de manière adaptée à la situation »(Dahm, 2013 : 91). On peut dès lors supposer que si l'élève parvient à mobiliser à la fois ses savoirs, ses savoir-faire ainsi que ses savoir-être de manière à établir des liens cognitifs, linguistiques et pragmatiques entre différentes langues, il acquiert une compétence plurilingue.

Dans le présent article, nous nous attacherons à examiner les liens cognitifs et linguistiques que l'élève établit entre les différentes langues appartenant à son répertoire, lors d'activités de réflexion métasémantiques inscrites dans des séances d'approches plurielles fondées sur des langues inconnues (APLI). Ce répertoire est restreint au français L1 et à l'anglais L2 (certains élèves étudient également le latin), puisque la cohorte étudiée est composée de quatre-vingt huit élèves de niveau cinquième, parfaitement monolingues dans leur environnement familial (informations obtenues dans le questionnaire pré-expérimentation). Nous explorerons donc la manière dont les élèves prennent appui sur leurs savoirs et savoir-faire afin de résoudre le problème d'accès au sens auquel ils sont confrontés.

Nous émettons l'hypothèse selon laquelle un dispositif didactique s'appuyant sur plusieurs langues inconnues, mis en œuvre de manière systématique et régulière, aide l'élève à développer sa compétence métalinguistique, compétence transversale que nous supposons indépendante du répertoire langagier utilisé. La mise au contact de langues inconnues, sans objectif d'enseignement/apprentissage, permet à l'élève de se décentrer d'un objectif d'appropriation de connaissances : sa confrontation à cet obstacle déclenche, par conséquent, la conscientisation des savoirs et savoir-faire acquis de manière antérieure à la séance d'APLI.

Neuf séances d'APLI furent menées sur l'année 2011-2012 : trois séances en néerlandais, trois en italien et trois en finnois. Les élèves ont été amenés, par groupes de quatre, à effectuer tour à tour une réflexion métasémantique, métasyntaxique et métaphonologique et ce, pour chacune des langues. Nous avons cherché à com- 
prendre, en nous appuyant sur les travaux de John R. Anderson (1996), comment les élèves sont amenés à repérer des solutions reconnues pertinentes en L1 et en L2, par la mobilisation de leurs savoirs déclaratifs et procéduraux. Ce repérage devrait leur permettre de construire un opérateur pour résoudre le problème auquel ils sont confrontés dans la L3 qu'ils découvrent.

Après avoir défini les concepts théoriques nécessaires à l'interprétation et proposé un schéma décrivant les processus cognitifs mis en jeu lors des APLI, nous préciserons la constitution du corpus. Nous pourrons dès lors présenter les résultats et mener la discussion en conséquence.

\section{Cadre théorique}

Examiner les liens cognitifs établis par les élèves, peut se faire en prenant appui sur la théorie de l'apprentissage telle que définie par Anderson (1983). Par conséquent, seront étudiés les concepts de connaissances déclaratives et procédurales, de mémoire déclarative et procédurale ainsi que de savoirs explicites et implicites.

\subsection{Connaissances déclaratives et connaissances procédurales}

La théorie de l'apprentissage proposée par Anderson (1983) repose sur la distinction classique en psychologie cognitive entre « un savoir déclaratif, constitué d'un assemblage d'informations indispensables pour générer une action, et un savoir procédural constitué de procédures de mise en œuvre de l'action » (Griggs et al. $2002: 18)^{1}$. Pour Anderson (1987: 206), une des caractéristiques de base du système déclaratif est qu'il n'est pas nécessaire de connaître l'utilisation du savoir pour l'emmagasiner. Il est donc facile de mettre un savoir pertinent dans le système alors qu'un effort considérable doit être fait pour convertir ce savoir en comportement.

Anderson $(1980,1983)$ découpe le processus d'acquisition en trois phases : la première qu'il appelle 'déclarative' (ou 'cognitive'), la deuxième 'associative' et la dernière 'procédurale'.

La première phase implique la compilation de connaissances déclaratives, ce qui requiert toujours un effort. La connaissance déclarative est un savoir dont l'apprenant a conscience ; il peut donc en parler. Pour Rebecca Oxford (2011 : 50), ce sont soit des connaissances sémantiques qui incluent des faits, des concepts, des noms, des dates ou des règles ou alors il s'agit de connaissances épisodiques (basées sur le souvenir d'un événement). Les apprenants organisent et représentent mentalement les connaissances déclaratives sous forme de schèmes qui peuvent aisément être oubliés dès lors qu'ils ne sont plus pratiqués (Oxford, 2011).

\footnotetext{
${ }^{1}$ Afin d'éviter les confusions entre les 'savoirs' qui constituent l'une des ressources participant au développement d'une compétence et le 'savoir déclaratif' tel que défini par Anderson (1983), nous choisissons d'utiliser les termes 'connaissances déclaratives' (qui désigne "les savoirs, verbalisables » (Sagnier, 2012 : 409)) et 'connaissances procédurales' (ou « savoir-faire, stockés sous forme de règles de production [...] et difficilement verbalisables » (ibidem)) lorsque notre focalisation porte sur les processus d'apprentissage.
} 
Lors de la deuxième phase, les apprenants construisent des schèmes plus élaborés. Ils sont en mesure de détecter et d'éliminer les erreurs intégrées lors de la première phase et les associations sont renforcées par la pratique (Anderson, 1980). Les nouveaux apprentissages sont davantage assimilés par l'apprenant mais ne sont pas encore automatisés.

Ce n'est que lors de la troisième phase dite procédurale que les connaissances, jadis conscientes et soumises à l'effort, deviennent totalement instantanées, inconscientes. Michel Paradis (2009) considère la procéduralisation comme le remplacement progressif de l'utilisation de connaissances explicites lors de la construction de phrases, ce qui peut être réalisé grâce à l'utilisation d'une compétence implicite nouvellement acquise (de manière indépendante).

Dans la mesure où notre expérimentation ne porte pas sur l'acquisition d'une langue, mais uniquement sur la réflexion métalinguistique à partir de langues inconnues, on peut considérer la première phase comme étant essentielle. Pour Paradis (ibid.), au cours de la phase cognitive, l'apprenant procède à un encodage déclaratif : des faits importants pour l'habileté à construire sont mis en mémoire. Ils sont utilisés, grâce à des procédures interprétatives générales comme la construction d'analogies, pour construire un comportement cohérent et approprié. La condition est que le fait décrit puisse être mis en relation avec le problème actuel par un travail cognitif. Ce travail cognitif doit montrer le lien entre les deux problèmes et permettre de dégager les éléments pertinents.

\subsection{Mémoire déclarative et mémoire procédurale}

L'élève peut tout à fait stocker des informations relatives au fonctionnement de sa L1 (l'apprentissage de concepts grammaticaux, par exemple) dans sa mémoire déclarative mais ce n'est que par la mise en œuvre de processus explicites que ces connaissances seront réellement acquises. Oxford (2011: 281) désigne par mémoire déclarative, "la mémoire des faits ou des événements, non encore automatisés ou rendus habituels $»^{2}$ alors que pour cette auteure, la mémoire procédurale est « la mémoire du 'comment' (comment accomplir quelque chose) sans connaissance déclarative consciente $»^{3}$. Par conséquent, l'apprenant ne pourra transférer les informations stockées dans sa mémoire déclarative vers sa mémoire procédurale que si on l'incite à établir des règles procédurales par lui-même. Il s'agit, pour Danièle Chini (2008 : 62), de la construction « d'une mémoire procédurale [...] où les savoirs pré-existants sont sollicités de manière dynamique et opératoire et où les savoirs construits sont des schémas d'action ». On peut, dès lors, supposer que l'observation d'une langue inconnue, sous l'effet d'un effort attentionnel, permettra à l'élève de recourir à des savoirs (qu'il a de la L1 et/ou de la L2) stockés dans sa

\footnotetext{
2 Notre traduction de : «memory of facts or events, not yet automated or habitualized» (Oxford, $2011: 281)$.

${ }^{3}$ Notre traduction de : « memory of 'how to' (how to accomplish something) without conscious declarative knowledge » (Oxford, $2011: 295)$.
} 
mémoire déclarative qu'il sera amené à transformer en savoir procédural permettant de décoder le système linguistique en présence. Or, même s'il parvient au résultat escompté, le savoir peut demeurer implicite.

\subsection{Savoir explicite et savoir implicite}

Pour Rod Ellis (2008 : 962), « le savoir explicite en L2 est la connaissance des règles et des objets qui existent dans une forme analysée afin que les apprenants soient en mesure de rapporter ce qu'ils savent $»^{4}$. Cet auteur donne la définition suivante du savoir implicite (ibid. : 965$)^{5}$ :

«Le savoir implicite d'une langue est une connaissance qui est intuitive et tacite. Elle ne peut pas être directement signalée. [...]. L'étude de la compétence linguistique est l'étude de la connaissance implicite d'un locuteur-auditeur $»$.

Cette distinction savoir implicite/savoir explicite apparaît comme fondamentale pour notre recherche. La plupart des individus ont une connaissance implicite de la L1, si bien que les concepts propres à l'analyse linguistique, pourtant appris dans le cadre scolaire, demeurent souvent abstraits et restent confinés dans la mémoire déclarative. La réflexion métalinguistique permet de rendre explicites les savoirs et de participer à leur procéduralisation. Claus Faerch (1986) postule qu'il y a 'prise de conscience' lorsqu'il y a passage de la connaissance implicite vers l'explicite. Il parle d' 'automatisation' dès lors qu'il y a passage de la connaissance explicite vers la connaissance implicite. On peut également distinguer, de la même façon, l'enseignement/apprentissage implicite (sans prise de conscience) de l'enseignement/apprentissage explicite (avec prise de conscience).

Les très nombreuses recherches effectuées en psychologie de la cognition liée à l'acquisition de la L2 ont montré que le chemin le plus efficace pour maîtriser une L2 passe par une combinaison d'apprentissages explicites et implicites. Robert DeKeyser et Alan Juffs (2005) considèrent que la meilleure façon de développer des connaissances implicites/procédurales/automatisées est non pas de les fournir directement, mais de favoriser les conditions optimales pour leur acquisition au long terme en fournissant un point de départ explicite.

Or un élève en contexte scolaire ne saurait construire du savoir sur la L3 sans avoir au préalable des savoirs sur la L1 et/ou la L2 ainsi que sur les mécanismes du langage. La L1, en tant que langue de scolarisation, est le langage, le système sémantique de référence pour les échanges entre apprenants et enseignant. Elle sera éga-

\footnotetext{
${ }^{4}$ Notre traduction de "Explicit L2 knowledge is the knowledge of rules and items that exists in an analysed form so that learners are able to report what they know» (R. Ellis, 2008 : 962).

${ }^{5}$ Notre traduction de "Implicit knowledge of a language is knowledge that is intuitive and tacit. It cannot be directly reported. The knowledge that most speakers have of their L1 is implicit. The study of linguistic competence is the study of a speaker-hearer's implicit knowledge » (R. Ellis, 2008 : 965).
} 
lement la langue utilisée pour verbaliser les traces des opérations mentales en cours, lors de la pratique réflexive. Néanmoins, nous postulons que même si la L1 est l'instrument privilégié de la pratique réflexive, elle n'en devient pas pour autant la seule ressource langagière disponible. L'élève pourra tout autant s'appuyer sur ses connaissances du fonctionnement de la L2 afin d'émettre des hypothèses concernant la Ln inconnue.

\subsection{Schéma cognitif des processus activés lors des APLI}

Lorsque nous introduisons une démarche fondée sur notre approche plurielle, l'objectif n'est plus l'appropriation de la nouveauté mais essentiellement la mobilisation d'acquis antérieurs. Par conséquent, les schémas cognitifs que l'élève va activer seront différents de ceux activés lors de l'apprentissage de l'anglais L2, même si les phases demeurent les mêmes.

Analysons les processus cognitifs mis en jeu par chaque étape de la situation d'apprentissage propre aux APLI en prenant appui sur le classement des stratégies d'apprentissage effectué par Geoffrey Sockett (2001) :

- Lors de la phase cognitive (étape 1: comparaison ou traduction d'éléments linguistiques appartenant à leur répertoire plurilingue, recours aux stratégies d'inférence et de déduction pour élargir la compréhension), les élèves sont amenés à activer leurs connaissances antérieures, de manière explicite, et à prendre conscience des stratégies qu'ils mettent en œuvre. L'activation et la prise de conscience devraient leur fournir les ressources nécessaires pour parvenir à résoudre le problème d'accès au sens qui leur est proposé. Ils sont alors amenés à mobiliser à la fois des savoirs déclaratifs et procéduraux, et ce de manière consciente.

- Lors de la phase associative (étape 2, travaillée lors des séances d'activités métasyntaxiques), les élèves mettent en correspondance les savoirs déclaratifs et procéduraux avec la situation-problème de création de phrases nouvelles. On peut dès lors supposer que l'aspect procédural (implicite) des stratégies mises en œuvre prévaut, puisqu'il s'agit avant tout de parvenir à résoudre le problème auquel ils sont confrontés.

- Lors de la phase de procéduralisation (étape 3: transfert inconscient vers d'autres langues), l'élève détecte et élimine les erreurs afin d'automatiser les procédures. On parle alors de phase autonome. Cette phase n'est pas explicitement travaillée dans les APLI.

- La modélisation proposée ci-dessous (schéma 1) permet de souligner l'activation des savoirs qui se fait de manière consciente et qui contribue au développement de la compétence métalinguistique. Elle souligne également l'aspect spiralaire de l'activité d'APLI puisque l'on peut considérer la réflexion métalinguistique sous forme de spirale infinie, permettant la réactivation continue de savoirs antérieurs, améliorant très probablement la maîtrise de ces mêmes savoirs en L1 et/ou en L2. 


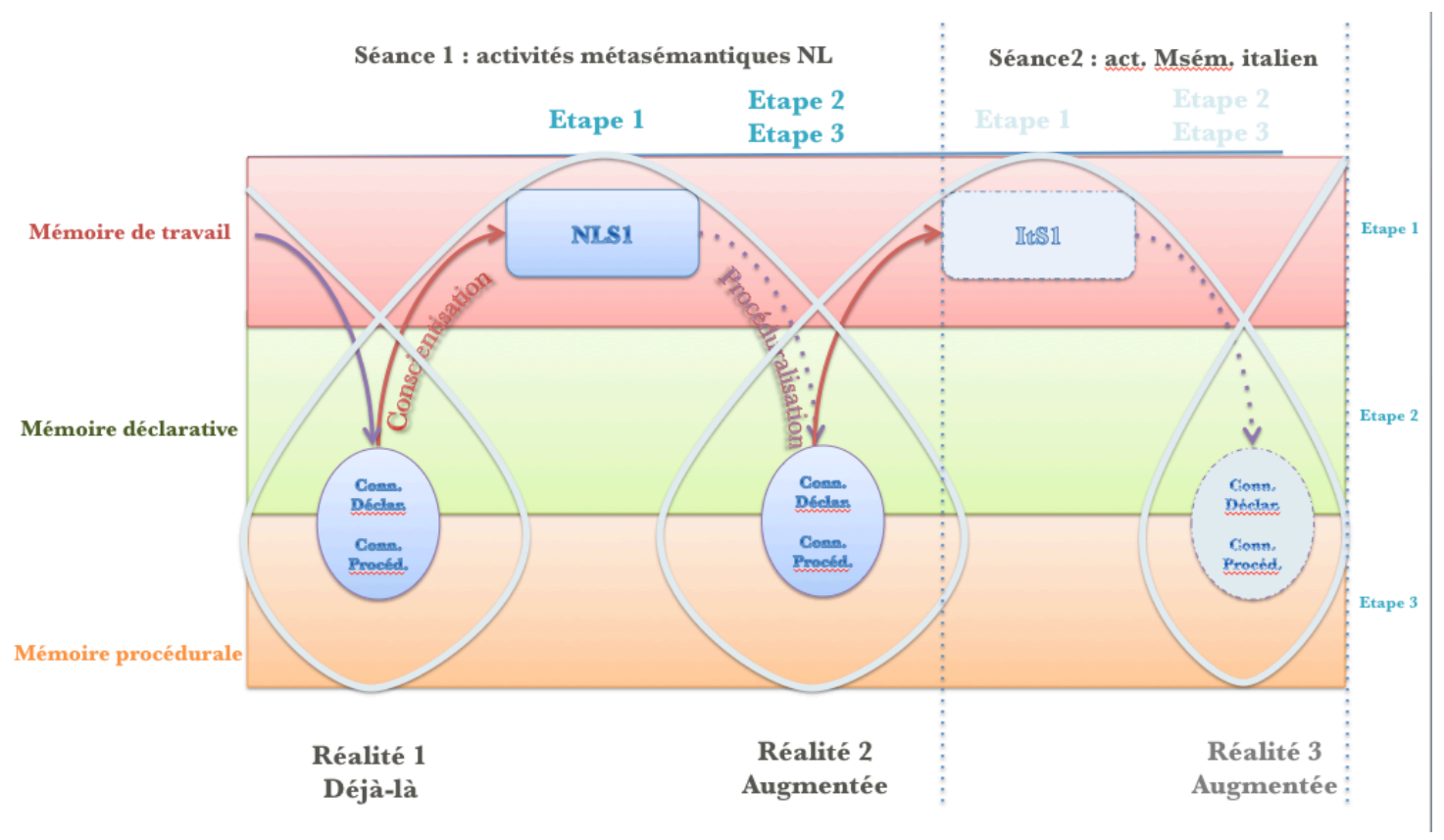

Schéma 1 : Modélisation (partielle) des processus cognitifs sous-jacents à la démarche d'APLI

Contrairement à l'enseignement de l'anglais qui prend appui sur les acquis antérieurs de manière implicite, la démarche d'APLI insiste avant tout sur la conscientisation des savoirs nécessaires à la résolution du problème. Par contre, les phases cognitive et associative ne bénéficient pas d'un accompagnement didactique dans le cadre des APLI : c'est la réflexion menée au sein des groupes qui leur permet d'activer ces connaissances.

\section{Cadre méthodologique}

\subsection{Choix pédagogiques propres aux APLI}

La première séance d'APLI, qui sert de support à cet article, s'appuie sur des activités métasémantiques, organisées de la même manière pour chacune des langues abordées : les élèves sont amenés à résoudre le problème d'accès au sens.

Les séances ont été menées par les enseignants de langue, sur un temps habituellement attribué à l'enseignement de l'anglais et prennent appui sur des textes abordant des sujets usuels de la classe d'anglais au collège. Le premier texte, en néerlandais, aborde la présentation de la famille (lettre écrite à un correspondant), le deuxième, en italien, est une devinette autour de la description de Mickey alors que 
le troisième, en finnois, parle des goûts et pratiques musicales ( $c f$. annexe 1 pour prendre connaissance des traductions) :

Texte $1:$ texte en néerlandais

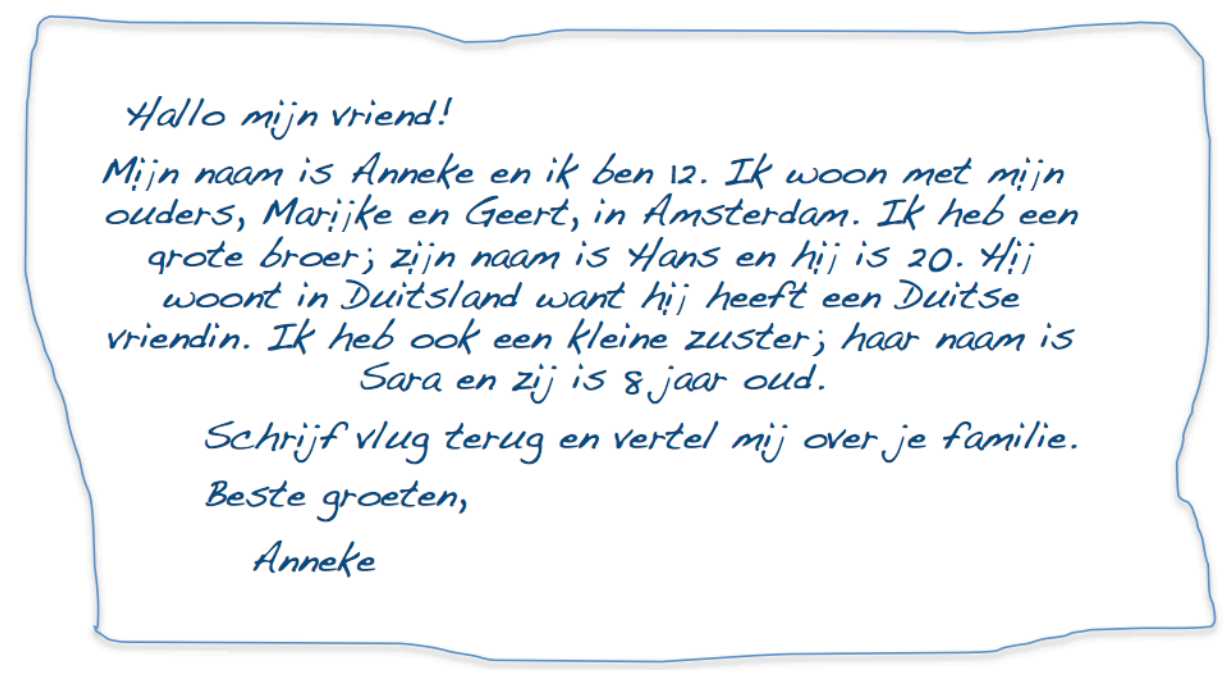

Texte en néerlandais : lettre écrite à un correspondant

Texte 2 : Texte en italien

"Sono un topo molto famoso. Io sono piccolo, con grandi orecchie nere.

Indosso pantaloni rossi con grandi bottoni bianchi. I miei migfiori amici sono

Paperino e Pippo e la mía Gella ragazza si chiama Minnie.

Mio padre è molto famoso: il suo nome è Walt Disney!

Chi sono io?"

Texte en italien : devinette portant sur Mickey 


\title{
Texte 3 : Texte en finnois
}

\author{
Päivi: "Rakastatko musiikkia?" \\ Timo: "Kyllä, minä rakastan. Lataan paljon musiikkia Internetistä. \\ Päivi: "Soitatko musiikkia?" \\ Timo: "Kyllä, minä soitan. Soitan pianoa. Sisareni Eija ei soita pianoa, mutta hän \\ soittaa kitaraa. Entä sisaresi Nina?” \\ Päivi: "Sisareni Nina rakastaa rap-musiikkia. Rakastaako sisaresi Eija myös rap- \\ musiikkia?"
}

Timo: "Ei, hän ei rakasta."

Texte en finnois : discussion autour de goûts musicaux

Cette première séance pour chaque langue inconnue ( $\mathrm{S} 1$, portant sur des activités métasémantiques), se déroule toujours de la même façon. Après un travail individuel de sept minutes permettant à l'élève de se concentrer sur la langue inconnue, s'ensuit le travail de groupe qui dure vingt-cinq minutes. Il s'agit d'indiquer les éléments ayant été compris et de justifier la manière dont cette compréhension a été rendue possible. Pour ce faire, une fiche de groupe comportant les consignes «Qu'avez-vous compris ? Comment avez-vous fait pour comprendre ? » fut complétée et rendue à l'enseignant. Lors de la phase de synthèse (de dix minutes), l'enseignant valide l'ensemble des réponses et guide les élèves vers une mise en relation avec les éléments pertinents issus de leur répertoire langagier (L1 et/ou L2).

\subsection{Constitution du corpus}

Dans la mesure où la population étudiée est strictement monolingue, l'interaction entre pairs au sein du travail de groupe ${ }^{6}$ permet d'accroître la mobilisation des acquis antérieurs, par le questionnement qu'elle suscite. La verbalisation de l'interaction existant au sein des différents groupes (vingt-deux au total) fut enregistrée et analysée afin de repérer des invariants dans les observations effectuées.

Le corpus étudié dans cet article est enrichi, pour chacune des séances portant sur des activités métasémantiques, des vingt-deux fiches de groupe complétées conjointement et répondant à des consignes précises.

\footnotetext{
${ }^{6}$ Des groupes hétérogènes, de sexe mixte comprenant chacun trois ou quatre élèves, ont été constitués par la chercheure, à partir de pré-tests linguistiques et d'un questionnaire pré-expérimentation.
} 


\subsection{Méthodologie d'analyse}

Nous avons choisi de mener une analyse qualitative, de manière à pouvoir comprendre les processus cognitifs sous-jacents à la résolution du problème d'accès au sens inscrit dans la démarche d'APLI.

Les élèves sont amenés à solliciter leurs connaissances déclaratives et procédurales tant en L1 qu'en L2 afin de construire, de transformer et d'appliquer les nouvelles connaissances obtenues en L3.

La mobilisation des connaissances déclaratives sera étudiée en analysant les processus cognitifs inhérents à la mise en œuvre de la stratégie de comparaison et/ou de traduction. L'analyse des stratégies d'inférence et/ou de déduction utilisées permettra de comprendre comment les connaissances procédurales sont mobilisées pour faciliter l'accès au sens. De plus, nous chercherons à connaître la manière dont les savoirs construits sont transformés en schémas d'action en étudiant l'orientation du traitement (de type ascendant ou descendant) inhérent à la stratégie de déduction.

\section{Résultats et discussion}

Lors de cette expérimentation, nous cherchons à analyser des pratiques automatisées que, d'ordinaire, les élèves ne verbalisent pas : il s'agit de savoir-faire procéduraux, implicites, que les apprenants mettent en œuvre pour accéder à la compréhension d'un texte en langue inconnue. Or, par le biais de la consigne «Comment avez-vous fait pour comprendre ? ", ces savoir-faire procéduraux sont amenés à la conscience par la focalisation de l'attention sur quelques points spécifiques. L'élève est alors amené à mobiliser ses connaissances déclaratives et, si possible, ses connaissances procédurales. Or, tant les connaissances déclaratives que procédurales peuvent devenir fossilisées, si elles ne sont pas utilisées de manière active. L'objet de cette expérimentation est d'amener certains savoirs et savoir-faire à la conscience, de les encoder sous forme de savoirs procéduraux puis, par un travail conscient, d'accompagner les élèves dans la construction d'une mémoire procédurale. Il s'agit, par conséquent, comme le pose Chini (2008) de solliciter de manière dynamique et opératoire les savoirs pré-existants (appartenant au répertoire plurilingue) et de transformer les savoirs construits en schémas d'action. La mémoire procédurale est, pour Oxford (2011), la mémoire du 'comment', qui ne nécessite pas de connaissances déclaratives conscientes.

\subsection{Mobilisation de connaissances déclaratives}

Lorsque l'élève essaie d'accéder au sens de la langue inconnue, il peut exprimer son appui sur un répertoire plurilingue et prendre conscience du soutien qu'apporte la connaissance de la langue anglaise (ou d'autres langues appartenant à leur répertoire plurilingue) à cette démarche. L'élève peut alors utiliser une stratégie de comparaison afin de comparer des langues entre elles, ou de traduction pour établir 
une équivalence immédiate entre items. Ces deux stratégies mobilisent des connaissances déclaratives, puisque l'élève peut en parler.

L'analyse qualitative des fiches de groupe fait apparaître une utilisation préférentielle de la stratégie de comparaison et/ou de traduction. Or, la mobilisation des connaissances peut-être soumise à des savoir-faire ou à des savoir-être.

\subsubsection{Effet des savoir-faire sur la mobilisation de connaissances décla- ratives}

Tous les élèves établissent, de manière conforme aux attentes, des comparaisons avec les langues appartenant à leur répertoire plurilingue, c'est à dire le français et l'anglais, et ce selon la proximité typologique pour le néerlandais et l'italien. Il est arrivé, à quelques rares reprises, que des élèves établissent des comparaisons avec des langues autres que le français L1 ou l'anglais L2. Il s'agit du latin, étudié en classe par un certain nombre d'élèves (répartis de manière équivalente dans les groupes) et de l'espagnol, langue pourtant non abordée en classe de cinquième.

Lors de la séance portant sur l'italien, deux groupes ont effectué une comparaison avec le latin et deux autres groupes ont effectué une comparaison avec l'espagnol. Les élèves ont très probablement relevé l'origine romane des langues et effectuent des parallèles en ce qui concerne le lexique, même s'ils n'ont pas encore étudié l'espagnol. Dans un des cas, la référence est exacte (mais non explicitement mentionnée) :

Extrait 1 : extrait de la fiche de groupe de ItS1 A3

\begin{tabular}{|c|c|}
\hline Information trouvée & Comment avez-vous fait pour la comprendre? \\
\hline grandi>grand & C'est thes Pogique - can sa ressemble ou fror: ais \\
\hline pantaloniziton & idem ... \\
\hline $\begin{array}{l}\text { ressi, bianchi } \\
\text { rove beins. }\end{array}$ & iderm et ga ressemble a l'espagnop \\
\hline
\end{tabular}

Extrait de la fiche du groupe A3 rédigée lors de la séance ItS1 ${ }^{7}$

Les élèves attestent de leur conscience de l'existence des familles de langues mais l'absence d'exemple montre qu'ils n'ont pas de connaissance précise de l'espagnol. Un autre groupe a établi le lien suivant, inexact :

Extrait 2 : extrait de la transcription de ItS1 A5

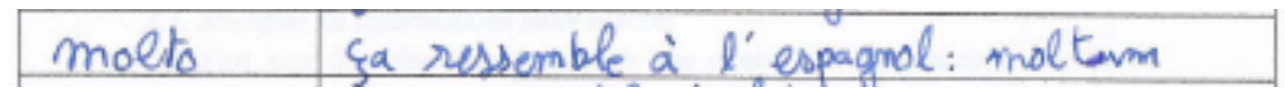

Extrait de la fiche du groupe A5 rédigée lors de la séance ItS1

\footnotetext{
${ }^{7}$ ItS1 désigne la séance d'activités métasémantiques menées à partir du texte en italien.
} 
Dans les deux cas, on peut confirmer la capacité de ces élèves à repérer des familles de langues et à effectuer des comparaisons en conséquence. Ils s'appuient non seulement sur leur connaissance d'items lexicaux épars mais ils sont également capables de les regrouper sous forme de familles de langues. Plusieurs groupes ont également repéré la ressemblance entre le néerlandais et l'allemand (pourtant non étudié). On peut supposer que ce savoir-faire contribue à leur aptitude à établir des liens afin de faciliter l'accès au sens. Il pourrait, en outre, contribuer à une attitude d'ouverture aux langues nouvelles.

\subsubsection{Effet des savoir-être sur la mobilisation des savoirs}

Même si la plupart des élèves ont rarement émis un jugement quant à la difficulté d'accès du texte, et ce quelle que soit la langue, un groupe a émis une opinion dès le début de l'interaction. Dans l'exemple ci-dessous, le groupe A5 aborde la séance d'activités métasémantiques portant sur l'italien de manière négative (extrait 3) :

\section{Extrait 3 : extrait de la transcription de ItS1 A5}

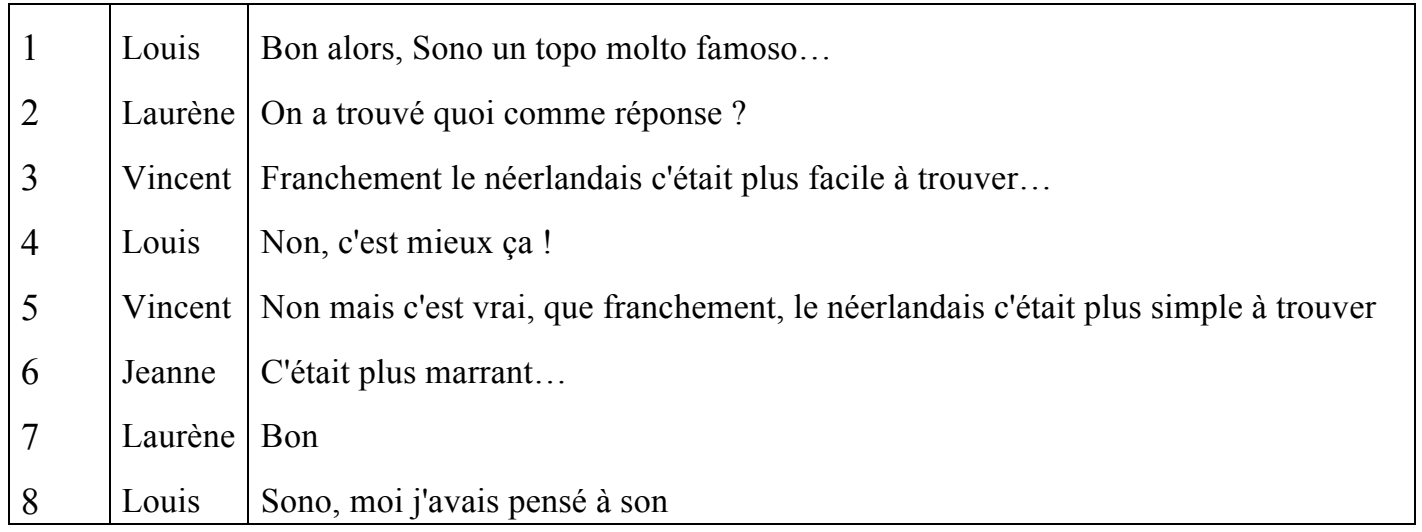

Transcription partielle de la discussion menée par le groupe A5 lors de la séance ItS1

Même si Louis émet un jugement de valeur positif, Vincent et Jeanne semblent plutôt réticents et comparent la difficulté d'accès au sens présenté par l'italien à celle du néerlandais. Laurène recentre l'attention par une simple interjection (1. 7 : «Bon ») et l'activité reprend son cours. Ensuite, le travail se poursuit, sans nouvelle référence à l'éventuelle facilité ou difficulté d'accès au texte. Les élèves ont pourtant beaucoup de difficultés à trouver du sens au texte, puisque seuls quatre mots sont repérés (extrait 4) :

Extrait 4 : extrait de la fiche de groupe de ItS1 A5 


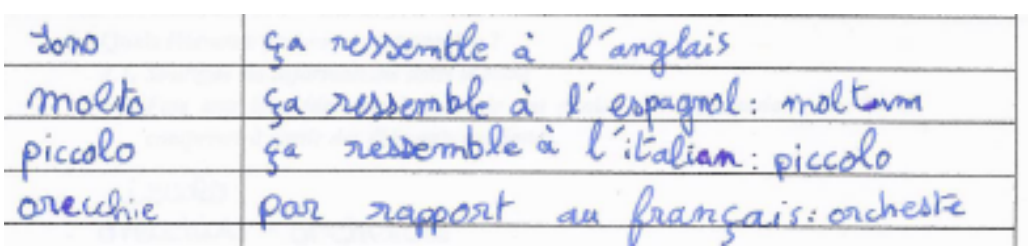

Extrait de la fiche du groupe A5 rédigée lors de la séance ItS1

On constate, grâce à leur fiche de groupe (extrait 4), que même s'ils ont mis en œuvre une stratégie de comparaison avec diverses langues, aucune association langue-source/langue-cible n'est valable. Ils se sont avant tout appuyé sur la forme du mot plutôt que sur le sens (extrait 5) :

\section{Extrait 5 : extrait de la transcription de ItS1 A5}

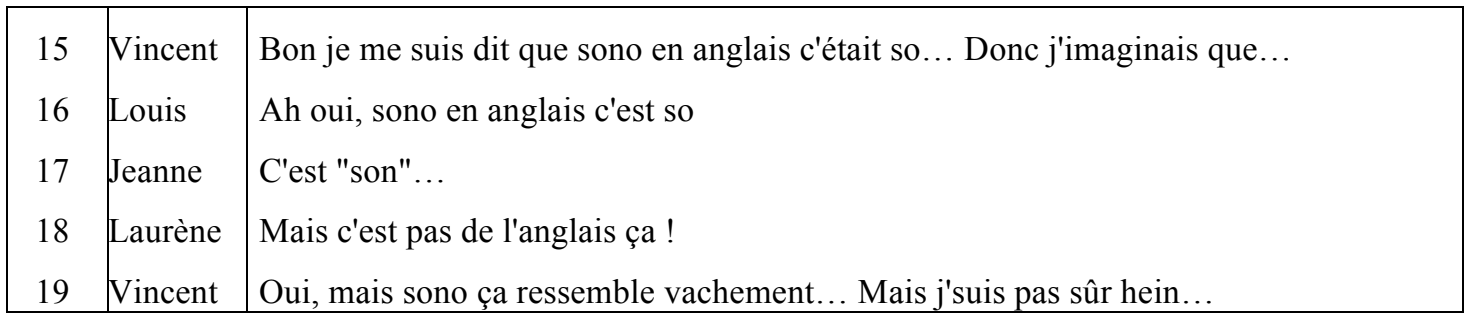

Transcription partielle de la discussion menée par le groupe A5 lors de la séance ItS1

Ils n'effectuent aucune réflexion quant à la signification des mots, que ce soit au niveau de la langue-source ou de la langue-cible. Les élèves ne parviendront pas à accéder au sens, alors qu'ils ont réussi à mobiliser des savoirs déclaratifs. Jeanne, dans l'avant dernier tour de parole de l'interaction enregistrée, exprime son désarroi : « Si ça se trouve ils nous ont mis un texte où ils ont tapé n'importe quoi... » (l. $60)$.

\section{Discussion}

Mettre en œuvre une stratégie de comparaison ou de traduction revient à prendre appui sur des connaissances déclaratives. Or, comme le pose Anderson (1987 : 206), une telle connaissance est aisément stockée dans notre système.

On peut dès lors concevoir que la mobilisation de savoirs déclaratifs, dans le cadre d'une stratégie de comparaison, soit le processus le plus immédiatement utilisé par les élèves : il requiert simplement une prise de conscience permettant d'établir la relation entre deux items. Cette prise de conscience permet de mettre le fait décrit 
en relation avec une solution reconnue comme pertinente et stockée dans la mémoire déclarative. Néanmoins, il ne suffit pas de mobiliser une connaissance déclarative pour parvenir à un résultat et obtenir un accès au sens; il est nécessaire que des relations de sens soient établies.

Lorsque les élèves parviennent à regrouper des langues sous forme de familles (extrait 1), on peut considérer qu'ils mobilisent à bon escient non seulement leurs savoirs sur l'existence de familles de langues mais également leur savoir-faire en ce qui concerne la maîtrise des démarches de comparaison. Cette mobilisation ne peut être efficace que si elle est combinée à des savoir-être, c'est-à-dire à une attitude d'ouverture.

Afin d'éviter l'interférence avec les préjugés linguistiques des élèves, la décision avait été prise de ne pas communiquer, en amont, la nature de la langue présentée. Les seules remarques subjectives exprimées par les élèves proviennent donc réellement du ressenti lié à l'observation de la langue en présence, et non pas d'éventuelles idées préconçues concernant les langues 'faciles' ou 'difficiles'. Or, il semblerait que le jugement porté par l'apprenant sur les ressemblances générales entre les langues a un effet facilitateur général sur les processus de compréhension, conformément à l'hypothèse de Hakan Ringbom (2002).

Cette hypothèse se vérifie lorsqu'on compare les interactions orales et les fiches du groupe A5, pour les trois langues. Une écoute des enregistrements des séances de néerlandais et de finnois fait état d'une absence de jugement de valeur sur les langues abordées. Lorsqu'on examine la fiche de ce groupe portant sur l'accès au sens du texte en néerlandais (cf. annexe 2), on constate que les élèves sont parvenus à comprendre une grande partie du texte, même s'ils ne déclarent qu'un appui sur des stratégies de comparaison. Lors de la séance de finnois (annexe 3), ils sont parvenus à établir des comparaisons entre le français et le finnois, à partir des internationalismes. Il semblerait donc, que pour ce groupe, le jugement négatif porté sur l'italien ait eu un impact sur leur aptitude à accéder au sens du texte ; on peut donc supposer qu'ils n'ont pas réussi à atteindre le niveau général de transfert, ou premier niveau, tel que défini par Ringbom (2002).

L'analyse de l'interaction du groupe A5 lors des séances d'activités métasémantiques a permis de mesurer l'effet de la psychotypologie ${ }^{8}$ (Kellerman, 1979) sur l'aptitude des élèves à mobiliser les savoirs nécessaires à la mise en œuvre d'une stratégie efficace. Nous rejoignons Marie-Christine Deyrich (2007) pour qui l'évaluation subjective de la distance typologique entre les langues modifie le comportement des apprenants dans les transferts lexicaux. Il s'agit bien d'une 'évaluation subjective' : dans le cas présent, une telle anticipation n'avait pas été réalisée par le groupe de recherche puisque la notion de proximité linguistique semblait prévaloir. Une possible réaction subjective était envisagée pour la séance portant

${ }^{8}$ Pour Kellerman (1979), la psychotypologie désigne la manière dont un apprenant envisage la proximité linguistique. 
sur le finnois, or les groupes n'ont émis aucun jugement de valeur lors de cette séance. L'écoute de toutes les interactions (vingt-deux pour chacune des séances) permet d'affirmer que les élèves, en dehors de quelques amusements liés à une projection de l'oral, n'émettent pas de jugement de valeur concret sur la 'facilité' ou la 'difficulté' d'accès d'une langue. Ils s'émerveillent parfois devant leur aptitude à comprendre des langues inconnues, mais sont essentiellement absorbés par la tâche et par la résolution du problème présenté. L'exemple du groupe A5 présenté ci-dessus est unique. Il nous permet de supposer que la psychotypologie a non seulement un effet sur les transferts linguistiques, mais qu'elle pourrait également avoir un effet sur la mise en œuvre de stratégies d'apprentissage. Cette évaluation subjective impacte le premier niveau de transfert (ou niveau général) entre languesource et langue-cible défini par Ringbom (2002).

Le deuxième niveau ou niveau 'item' est associé aux processus établissant des identifications interlinguistiques, élément par élément. On constate, en effet, que la plupart des groupes établissent une relation un à un entre les items individuels en faisant correspondre le sens d'un mot en Ln avec celui d'un mot en L1. Dans la mesure où ils n'ont pas de connaissances de la langue cible, ils identifient les ressemblances entre les items en s'appuyant davantage sur la forme que sur le sens. C'est la raison pour laquelle le groupe A5 a effectué les appariements erronés entre les mots issus des deux langues. On peut donc supposer que la psychotypologie peut également affecter le deuxième niveau de transfert.

Le troisième niveau ou le niveau 'système' se rapporte aux processus durant lesquels l'apprenant identifie une identité de sens entre des items, et pas nécessairement de forme. La seule stratégie de comparaison entre deux langues ne permet pas de directement identifier le sens entre deux items ; pour qu'une telle identification ait lieu, une stratégie d'inférence ou de déduction semble nécessaire. Très probablement, seule une évaluation subjective positive des langues en présence permet d'atteindre le troisième niveau. Il s'agit, dès lors, de mobiliser des savoirs procéduraux.

\subsection{Mobilisation de connaissances procédurales}

Lorsqu'on prend appui sur la théorie d'Anderson (1980), il est usuel d'opposer le connaissances déclaratives (ou savoir 'que') aux connaissances procédurales (ou savoir 'comment'). Les connaissances procédurales désignent les savoirs qui peuvent être utilisés de manière automatisée, non consciente. (Ellis, 2008 : 976). Griggs et al. (2002) considèrent que les connaissances procédurales sont constituées de procédures de mise en œuvre de l'action. Il s'agit donc de comprendre comment l'élève parvient à mobiliser ses connaissances sur le fonctionnement des langues pour résoudre son problème d'accès au sens. L'objet de notre réflexion portera, ci-dessous, sur la mobilisation de concepts ou de règles grammaticales permettant d'affiner la compréhension. 


\subsubsection{Mobilisation de connaissances procédurales de manière impré- cise}

L'examen des fiches de groupe montre une difficulté pour les élèves à mobiliser des connaissances procédurales, puisqu'elles requièrent une réelle maîtrise des concepts avec une capacité de distanciation par rapport à des savoirs d'ordinaire automatisés. On constate cependant une évolution dans les fiches de groupe, au fur et à mesure des langues présentées : lors de la première séance d'APLI portant sur le néerlandais, aucun groupe ne fait état d'une réflexion prenant en compte le fonctionnement des langues (que ce soit la langue-cible ou la langue-source). Lors de la deuxième langue étudiée (l'italien) un groupe (E5) relève les éléments suivants (extrait 6) :

Extrait 6 : extrait de la fiche de groupe de ItS1 E5

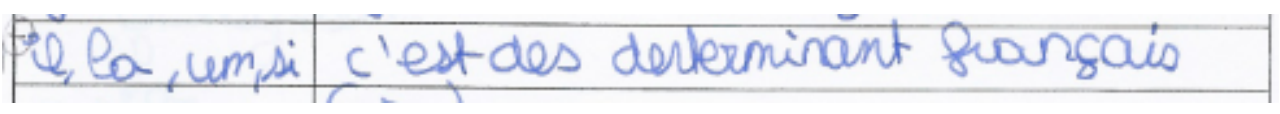

Extrait de la fiche du groupe E5 rédigée lors de la séance ItS1

Même s'ils sont parvenus à identifier une catégorie grammaticale, on ne voit pas d'utilisation directe du concept pour accéder au sens. Le savoir demeure donc davantage déclaratif que procédural.

Lorsqu'ils observent le finnois, plusieurs groupes relèvent la catégorie grammaticale 'verbes' pour justifier les multiples occurrences des formes conjuguées de rakastan et de soitan. La formulation peut rester imprécise, comme nous le montre l'exemple ci-dessous (extrait 7) :

Extrait 7 : extrait de la fiche de groupe de FinnS1 A6

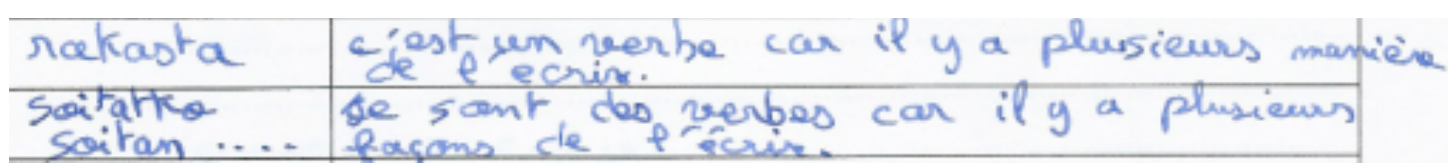

Extrait de la fiche du groupe A6 rédigée lors de la séance FinnS1

Le groupe A6 a repéré l'existence de verbes mais ne parvient pas à utiliser une métalangue appropriée. Néanmoins, la mobilisation de ce savoir procédural leur a permis de comprendre les occurrences différentes des verbes.

Un groupe est cependant capable de mobiliser ses savoirs procéduraux avec précision (extrait 8) :

Extrait 8 : extrait de la fiche de groupe de FinnS1 C5 


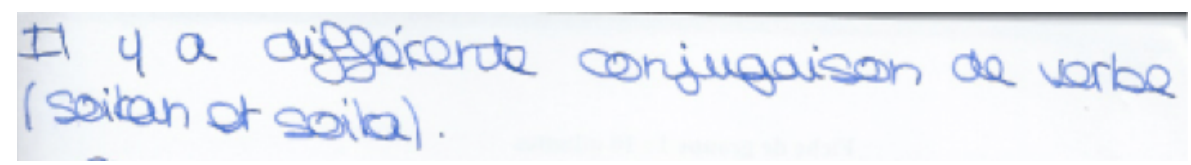

Extrait de la fiche du groupe C5 rédigée lors de la séance FinnS1

On remarque l'utilisation d'une métalangue précise, même s'ils ne sont pas parvenus à analyser le fonctionnement de ladite conjugaison, ce qui leur aurait permis d'obtenir des éléments d'information complémentaires.

\subsubsection{Mobilisation de savoirs procéduraux pour accéder au sens}

C'est l'examen de la transcription de l'interaction du groupe A3 essayant de mener à bien sa résolution de problème d'accès au sens en finnois qui offre le plus d'exemples de mobilisation de savoirs procéduraux. Le tour de parole 25 (dans l'extrait 9) montre comment le recours à des concepts grammaticaux guide la compréhension :

\section{Extrait 9 : premier extrait de la transcription de FinnS1 A3}

\begin{tabular}{|l|l|l|}
\hline 24 & Gabriel & $\begin{array}{l}\text { Lataan, ça veut dire écouter ? } \\
\text { Non, parce que regardez, y a un point, donc, si il y a un point, ça veut dire } \\
\text { que le verbe est après le sujet, normalement. Ça peut pas être le verbe, y'a } \\
\text { pas de sujet avant. }\end{array}$ \\
\hline
\end{tabular}

Transcription partielle de la discussion menée par le groupe A3 lors de la séance FinnS1

Louis prend initialement appui sur sa connaissance procédurale de la construction d'une phrase en français. Or, après plusieurs échanges et étant parvenu à comprendre le sens d'un autre verbe, rakastaako, il précise sa pensée en prenant appui sur ses (faibles) connaissances en italien (extrait 10) :

Extrait 10 : deuxième extrait de la transcription de FinnS1 A3

\begin{tabular}{|c|c|c|}
\hline 75 & Morgane & Rakastaako musiikkia, ça c'est écoutes-tu de la musique? \\
\hline 76 & Louis & $\begin{array}{l}\text { C'est comme l'italien ou je sais plus quelle langue, il n'y a pas de détermi- } \\
\text { nant. }\end{array}$ \\
\hline 77 & Manon & Alors rakastatko, pour vous, c'est écouter de la musique? \\
\hline 78 & Louis & C'est comme l'italien, il n'y a pas de déterminant, bon après... \\
\hline
\end{tabular}

Transcription partielle de la discussion menée par le groupe A3 lors de la séance FinnS1 
Louis semble avoir établi un lien entre le fonctionnement de l'italien et le fonctionnement du finnois. Il est donc parvenu à utiliser ses savoirs procéduraux sur une des langues-source pour faciliter la compréhension. En l'occurrence, même si le savoir déclaratif est imprécis (utilisation du terme 'déterminant' en lieu et place de 'pronom personnel'), le savoir procédural lui permet de comprendre le fonctionnement du finnois. Quelques échanges plus tard, Manon prend appui sur sa connaissance du fonctionnement de la langue anglaise pour proposer une solution leur permettant de comprendre une difficulté (Extrait 11) :

\section{Extrait 11 : troisième extrait de la transcription de FinnS1 A3}

\begin{tabular}{|l|l|l|}
\hline 81 & Morgane & Lataan, on sait pas ce que c'est,... musique sur internet \\
82 & Manon & Je cherche sur internet... \\
83 & Louis & C'est peut-être un groupe de mots, un groupe nominal qui veut dire... \\
84 & Morgane & Sur $?$ \\
85 & Louis & Non, ça peut pas être sur musique internet \\
86 & Manon & $\begin{array}{l}\text { Mais non, c'est pas obligé...En anglais, tu sais, les mots ils sont inversés, } \\
\text { ça peut être... }\end{array}$ \\
\hline
\end{tabular}

Transcription partielle de la discussion menée par le groupe A3 lors de la séance FinnS1

En soulevant la possibilité d'inversion de l'ordre des mots, Manon ouvre le champ de la compréhension. Finalement, le latin vient servir d'appui à la compréhension (extrait 12) :

Extrait 12 : quatrième extrait de la transcription de FinnS1 A3

\begin{tabular}{|c|c|c|}
\hline 99 & Louis & Rakastan, rakastaa, rakastatko... \\
\hline 100 & Manon & Est-ce que c'est une langue à déclinaison ou pas? \\
\hline 101 & Louis & On s'en moque \\
\hline 102 & Manon & $\begin{array}{l}\text { Non, ça peut être utile, savoir si c'est un verbe ou pas. S'il y a des décli- } \\
\text { naisons, on saura que les mots peuvent changer de terminaison }\end{array}$ \\
\hline 103 & Morgane & C'est quoi des déclinaisons? \\
\hline 104 & Manon & $\begin{array}{l}\text { Déclinaisons, c'est quand le nom change de terminaison suivant quelle } \\
\text { fonction il a, suivant si il est COD, complément d'objet... }\end{array}$ \\
\hline
\end{tabular}

Transcription partielle de la discussion menée par le groupe A3 lors de la séance FinnS1 
Dans l'extrait ci-dessus, Manon essaye d'expliquer au groupe que les savoirs procéduraux peuvent aider à la compréhension (1. 102). C'est la raison pour laquelle elle attire l'attention sur les variations de sisaresi et sisareni (extrait 13) :

Extrait 13 : extrait 4 de la transcription de FinnS1 A3

\begin{tabular}{|l|l|l|}
\hline 110 & Manon & Sisaresi, ça veut dire quoi ? \\
111 & Gabriel & Sisaresi, sisareni \\
112 & Morgane & Donc, il ou elle \\
113 & Louis & Non parce que Nina, Nina et sisaresi, sisareni \\
114 & Manon & Non, je pense qu'il a des terminaisons changées, donc il y a une déclinai- \\
& & son \\
\hline
\end{tabular}

Transcription partielle de la discussion menée par le groupe A3 lors de la séance FinnS1

Manon souhaite montrer au groupe que les noms sisaresi et sisareni ont le même sens, mais que leur graphie différente provient très probablement d'une fonction différente dans la phrase. Or, elle ne parvient pas à expliquer ce concept : le savoir est procédural, automatisé et nécessiterait davantage d'effort pour être véritablement conscientisé. Même si une prise de conscience de l'existence de déclinaisons a lieu, elle ne permet pas au groupe d'accéder au sens.

\section{Discussion}

Si l'élève parvient à établir des liens entre le fonctionnement de la langue observée et le fonctionnement des langues appartenant à son répertoire plurilingue, alors on peut considérer qu'il prend appui sur des connaissances procédurales issues de ce même répertoire plurilingue pour accéder au sens. Or, bien souvent, l'apprenant stocke des informations relatives au fonctionnement de sa L1 et/ou de sa L2 (l'apprentissage de concepts grammaticaux, par exemple) dans sa mémoire déclarative sans qu'une réelle procéduralisation ait lieu. Il peut ainsi connaitre des conjugaisons, stockées dans sa mémoire déclarative, sans être en mesure de les appliquer lorsqu'il rédige un texte. On peut considérer que c'est par la mise en œuvre de processus explicites, nécessitant la conscientisation de ces savoirs déclaratifs, que ces connaissances seront durablement acquises et pourront être transférées vers la mémoire procédurale. Il semble donc utile de permettre aux élèves de 'manipuler des langues' afin qu'ils se rendent compte par eux-mêmes qu'il existe, par exemple, des conjugaisons dans une langue inconnue, non enseignée : les accompagner dans une comparaison avec la langue-source étudiée (l'anglais, par exemple) leur permettrait de se rendre compte de l'importance de ce concept, et 
pourrait les accompagner progressivement dans la prise en compte des spécificités propres à chaque système linguistique.

On constate que la prise en compte de savoirs procéduraux, même si elle n'apporte pas de réponse directe au niveau du sens, permet aux élèves d'envisager les occurrences linguistiques de manière plus large (extrait 7 FinnS1 A6) : elle ouvre donc le champ des possibilités de compréhension. De plus, ce n'est qu'en mobilisant de tels savoirs que le troisième niveau de transfert, soit le niveau 'système' (tel que défini par Ringbom, 2002), pourra être atteint. A ce niveau, les élèves procèdent à des extensions sémantiques, dont on perçoit les prémisses dans les extraits 9 à 13 (FinnS1 A3). Ringbom affirme que le transfert de sens a lieu essentiellement à partir de la L1 à moins que le locuteur n'ait un excellent niveau de maîtrise de la L2. Le transfert 'système' serait davantage une manifestation de l'influence de la L1 plutôt que de l'influence d'une langue étrangère. Néanmoins, les observations menées ci-dessus ne nous permettent pas de confirmer la prédominance de l'influence de la L1. On constate, en effet, le recours à un répertoire plurilingue en évolution : le groupe A3 a mobilisé des savoirs issus des séances précédemment mises en œuvre en italien. Il est à rappeler que l'un des objectifs des activités métasyntaxiques proposées sur l'italien est la prise de conscience de l'absence du pronom personnel. Louis semble avoir intégré cette information et s'en sert pour élargir ses possibilités d'accès au sens du texte finnois. Manon procède de la même façon en s'appuyant sur ses connaissances balbutiantes en latin. Ce recours à des langues nouvellement inscrites dans leur répertoire plurilingue soulève la question du niveau de maîtrise des langues-source et cible.

Les observations effectuées ci-dessus semblent davantage confirmer les études menées par De Angelis (2005), Rivers (1979), Selinker et Baumgartner-Cohen (1995), qui considèrent que le transfert peut tout aussi bien provenir d'une L2 (ou Ln) que l'apprenant maîtrise peu. Pour De Angelis (2005: 34), un à deux ans d'instruction formelle sembleraient suffire pour affecter la production et le développement de la langue-cible de manière significative. D'après nos observations, il semblerait que ce soit moins le temps d'instruction que l'attention apportée à une telle instruction et la conscientisation en résultant qui pourrait influencer le choix de la langue-source.

C'est par le recours à des langues inconnues, diverses, donc en multipliant les occurrences d'observation que l'on pourra aider l'élève à transformer des savoirs déclaratifs en savoirs procéduraux. Nous soulignons ici l'importance de la dimension 'langues non enseignées' des approches plurielles envisagées. Il nous semble que l'élève développe d'autant plus facilement son savoir-faire réflexif qu'il est amené à comparer, avec l'aide de ses pairs, des langues ne présentant pas d'enjeu de mémorisation ou de résultat, ce qui peut être anxiogène. On peut supposer que la répétition de telles activités l'accompagne dans la procéduralisation de ses savoirs qu'il pourrait alors transférer à la langue faisant l'objet d'un enseignement. On peut donc considérer que l'ensemble des activités métalinguistiques proposées 
dans le cadre de cette expérimentation participe à la construction d'une mémoire procédurale où les savoirs pré-existants sont sollicités de manière dynamique et opératoire: les élèves semblent transformer les savoirs construits en schéma d'action.

Or, accroître la compréhension en transformant les savoirs construits en schémas d'action peut nécessiter un traitement de type bottom-up (de bas niveau) ou de type top-down (de haut niveau).

\subsection{Recours aux processus de haut niveau et de bas niveau}

L'apprenant peut recourir à des processus de haut niveau (top-down) lorsqu'il capitalise sur des informations connues permettant de comprendre le sens général du texte ou à des processus de bas niveau (bottom-up) lorsqu'il analyse chaque mot à la recherche de sens ou de caractéristiques grammaticales (Howard, 1985). Il ne s'agit pas tant de 'niveaux' de traitement de l'information mais davantage d'une indication du 'sens' dans lequel procède le décodage. Les textes en langues inconnues soumis aux élèves présentent à la fois des indices textuels (lettres majuscules, chiffres, ponctuation ainsi que des mots transparents) mais permettent également la mobilisation de processus de haut niveau (connaissances scolaires, connaissances du monde). Or, d'après certains chercheurs (Gernsbacher, 1990 ; Hansen \& Jensen, 1994 ; O’Malley \& Chamot, 1990), inter alii), il existe une « dépendance du bas niveau ${ }^{9}$ (Field, $2004: 365$ ) : les apprenants débutants en L2 utiliseraient plus volontiers des données perceptuelles, des petites unités de sens, sans pour autant parvenir à donner du sens à la globalité, lors des activités de compréhension. La recherche menée par Long (1989), sur des activités de compréhension orale, tendrait à montrer que les informations de haut niveau jouent également un rôle important, et ce quelque soit le niveau de l'apprenant. Il semble, par conséquent, intéressant de comprendre comment les groupes ont procédé lors de la déduction et de repérer le type de traitement de l'information mis en œuvre.

Les trois textes soumis contiennent à la fois des éléments permettant de mettre en œuvre une déduction de bas niveau, ascendante (majuscules, chiffres pour l'âge, ponctuation) ainsi qu'une déduction de haut niveau, descendante (connaissances scolaires : repérage du type de texte et de l'organisation textuelle et connaissances du monde : éléments de géographie, le monde de Walt Disney et pratiques musicales).

Voici un exemple type d'une déduction de bas niveau, ascendante, menée sur le néerlandais (extrait 14) :

\footnotetext{
${ }^{9}$ Notre traduction de Field $(2004: 365):$ « bottom-up dependency ».
} 
Extrait 14 : extrait de la fiche de groupe de NLS1 E4

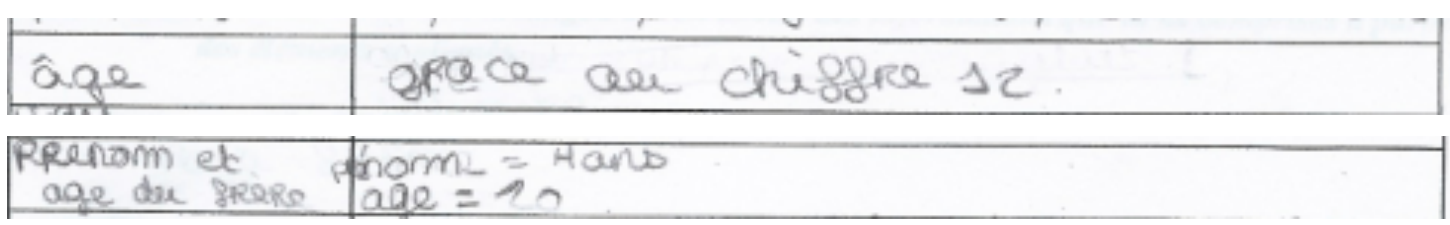

Extrait de la fiche du groupe E4 rédigée lors de la séance NLS1

Dans cet extrait, on peut observer comment les élèves ont su repérer l'âge (et exprimer leur prise de conscience) ainsi que le prénom du frère. Ils ont ainsi pu accéder à des informations basiques, néanmoins essentielles à la compréhension du texte.

Le recours à des processus de haut niveau est censé accroître la compréhension écrite. La compréhension du texte en italien est un support intéressant à l'observation du traitement de l'information de type descendant, puisqu'une telle réflexion, menée correctement, a pu tantôt aider les élèves dans leur compréhension, tantôt les induire en erreur.

\subsubsection{Recours aux processus de haut niveau, compréhension réussie}

Dans la fiche de groupe ci-dessous, on peut observer l'association de la couleur du pantalon aux connaissances que possèdent les élèves sur Mickey (extrait 15) :

\section{Extrait 15 : extrait de la fiche de groupe de ItS1 C6}

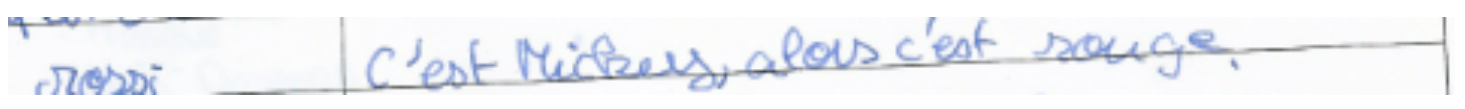

Extrait de la fiche du groupe C6 rédigée lors de la séance ItS1

Cette déduction de haut niveau a permis aux élèves d'éviter l'écueil de l'association de l'adjectif rossi avec la couleur rose, ce qui présentait une proximité graphique plus évidente. L'appui sur leurs connaissances du monde a permis une compréhension détaillée exacte.

\subsubsection{Recours aux processus de haut niveau, échec de la compréhen- sion}

Il arrive cependant que les connaissances du monde influencent négativement la compréhension. Lorsqu'on observe la fiche de groupe ItS1 A3, on constate que la 
déduction de haut niveau menée par le groupe les a induits en erreur et qu'il a eu un impact sur l'ensemble de la compréhension du texte (extrait 16) :

Extrait 16 : extrait de la fiche de groupe de ItS1 A3

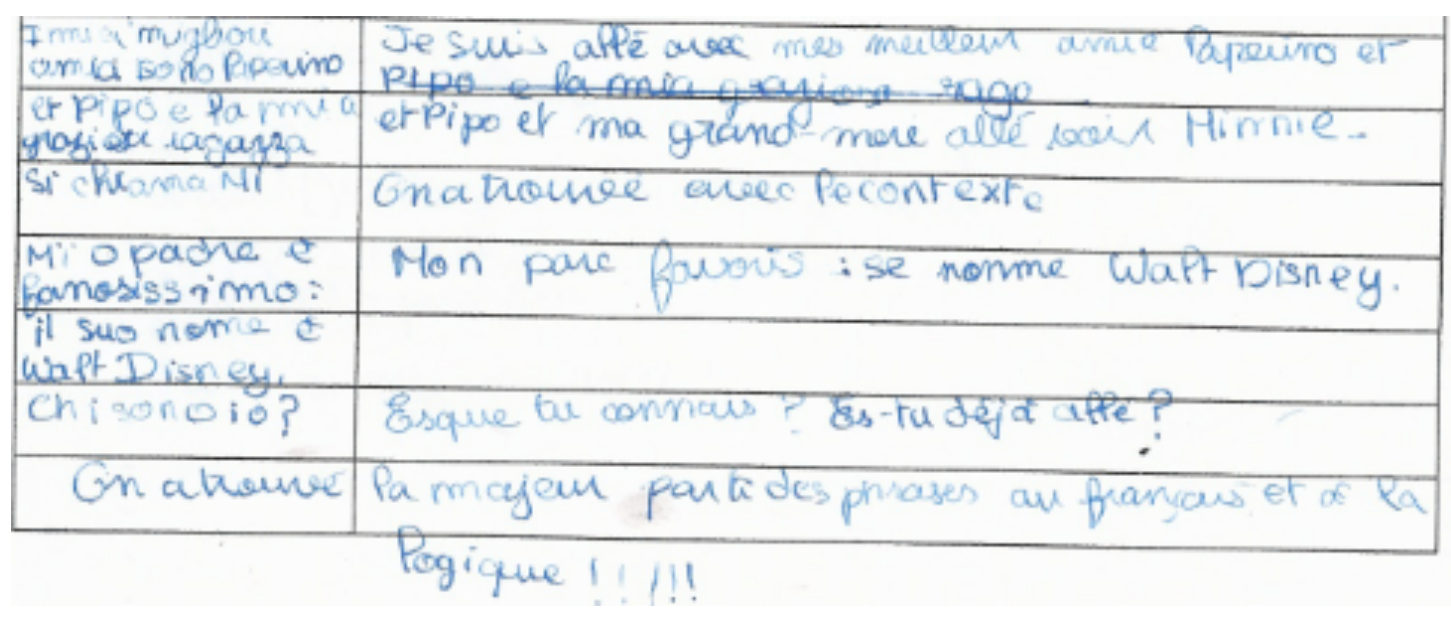

Extrait de la fiche du groupe A3 rédigée lors de la séance ItS1

Ce groupe déclare tout d'abord avoir utilisé une inférence pour comprendre la première phrase de cet extrait. Or, cette inférence a subi l'effet de la déduction de haut niveau, menée à bon escient, mais de manière erronée par le groupe. Ils ont, en effet, associé Walt Disney au parc d'attraction et ont, par conséquent, inféré la plupart des phrases à partir de cette déduction.

\section{Discussion}

Il semblerait, à première vue, que les élèves n'aient pas eu de difficultés pour mettre en œuvre des déductions, et ce quelle que soit l'orientation du traitement. La prise d'appui sur des indices textuels, associée à la comparaison avec les langues appartenant à leur répertoire plurilingue, a permis aux élèves de mettre en œuvre des stratégies d'inférence, ce qui les a amenés à comprendre le sens d'un grand nombre de phrases. Ils se sont également appuyés sur leurs connaissances scolaires, puisqu'ils ont été en mesure de procéder à des déductions de haut niveau à partir du type de texte proposé. Il est vrai que ceux-ci revêtent de grandes similitudes avec des activités proposées lors de l'apprentissage de l'anglais.

Or, dans l'extrait 16, la prise d'appui sur des processus de haut niveau (c'est à dire leur connaissance du monde de Walt Disney) a induit le groupe en erreur puisqu'ils 
associent le nom de Walt Disney au parc d'attractions. Cette déduction fausse ne fut pas anticipée par l'équipe de recherche : nous n'avions pas conscience de l'évolution des représentations liées à Walt Disney, désormais davantage connu pour le parc d'attraction que pour ses dessins animés.

On constate cependant que la majorité des déductions portant sur les autres langues ont été menées à bon escient et ont permis aux élèves d'améliorer leur compréhension de texte. Or, pour Cummins (1979), l'apprenant doit posséder un seuil minimal de compétence linguistique afin d'être en mesure d'utiliser des processus de haut niveau. Cette vision est confirmée par Clarke (1980) qui considère que l'attention des débutants est tellement focalisée sur le décodage qu'ils sont incapables de transférer vers la L2 (la Ln dans notre cas) les processus de haut niveau qu'ils mettraient naturellement en œuvre dans leur langue maternelle. On ne saurait pourtant affirmer, dans le cadre qui nous intéresse, que les élèves aient atteint un seuil minimal de compétence puisqu'ils observent des langues, par définition même, inconnues. Nos résultats qualitatifs semblent, par conséquent, contredire la théorie de la dépendance des processus de bas niveau et rejoindraient davantage la thèse de Stanovich (1980). Celui-ci considère que la relation entre les informations de haut niveau et de bas niveau est régulée par un mécanisme interactif-compensatoire. Les travaux de Perfetti (1985) viennent confirmer cette hypothèse puisqu'il a constaté que les informations de haut niveau sont utilisées de manière compensatoire, non pas pour renforcer le sens qui a déjà été dérivé d'un texte mais pour restaurer les parties qui n'ont pas été entièrement comprises.

\section{Conclusion}

Parvenir à comprendre un texte en langue inconnue ne saurait se faire sans la mobilisation à la fois des connaissances déclaratives et des connaissances procédurales. L'étude des fiches de groupe semble montrer que les élèves mobilisent le plus aisément des connaissances déclaratives, mais cette mobilisation peut être facilitée ou entravée par des savoir-faire ou des savoir-être.

Il apparaît que les stratégies de comparaison et de traduction naturellement mises en œuvre par les élèves s'appuient avant tout sur des connaissances déclaratives alors que la déduction et l'inférence nécessitent à la fois des connaissances déclaratives et procédurales. Contrairement à Ringbom (2002) qui considère que le transfert de sens a lieu essentiellement à partir de la L1, nous pensons que les élèves sont en mesure d'utiliser des connaissances récemment acquises pour élargir leurs possibilités d'accès au sens de la Ln, et rejoignons en cela les observations effectuées par De Angelis (2005). Il nous semble cependant que l'attention apportée à une telle instruction joue un rôle plus important que la durée d'instruction.

On peut donc affirmer qu'un dispositif didactique s'appuyant sur plusieurs langues inconnues, mis en œuvre de manière systématique et régulière, aide l'élève à développer sa compétence métalinguistique. Les activités métasémantiques proposées semblent offrir à l'élève la possibilité de développer sa compétence plurilingue. 


\section{Bibliographie}

Anderson, J. R. 1980. Cognitive psychology and its implications ((2000) 5th ed.). New York: Worth.

Anderson, J. R. 1983. The Architecture of Cognition. Cambridge, Mass. : Harvard University Press.

Anderson, J. R. 1987. « Skill Acquisition : Compilation of Weak-Method Problem Solutions ». Psychological Review, 94/2 : 192-210.

Beacco, J.-C. et al. 2010. Guide pour le développement et la mise en ouvre de curriculums pour une éducation plurilingue et interculturelle. Strasbourg : Conseil de l'Europe.

Candelier, M. 2003. L'éveil aux langues à l'école primaire. Evlang : bilan d'une innovation européenne. Bruxelles : De Boeck.

Castellotti, V. \& D. Moore. 2011. La compétence plurilingue et pluriculturelle: genèse et évolutions d'une notion-concept. In Blanchet, $\mathrm{Ph}$. et $\mathrm{P}$. Chardenet (dir.). Guide pour la recherche en didactique des langues et des cultures. Paris : Éditions des archives contemporaines, 241-252.

Chini, D. 2008. Objectif méthodologique: construction d'une mémoire procédurale? In Chini, D. et P. Goutéraux (dir.). Psycholinguistique et didactique des langues étrangères. Travaux du GEPED en hommage à Danielle Bailly. Paris : Ophrys, 55-67.

Clarke, M. 1980. « The short circuit hypothesis of ESL reading - or when language competence interferes with reading performance ». Modern Language Journal, $64: 203-209$.

Cummins, J. 1979. « Cognitive/academic language proficiency, linguistic interdependence, the optimum age question and some other matters ». Working Papers on Bilingualism, 19 : 197-205.

Dahm, R. 2013. Effets de l'introduction d'une approche plurielle fondée sur des langues inconnues sur le système didactique. Des éléments de cadrage à la mise en place expérimentale en classe d'anglais au collège. Thèse de doctorat. Université Bordeaux-Segalen, Bordeaux.

De Angelis, G. 2005. «Multilingualism and non-native lexical transfer : an identification problem ». International Journal of Multilingualism, 2/1 : 1-25.

DeKeyser, R., \& Juffs, A. (2005). Cognitive Considerations in L2 Learning. In Hinkel, E. (ed.). Handbook of Research in Second Language Teaching and Learning. Mahwah, NJ : Lawrence Erlbaum and Associate : chapter 24 (ebook).

Ellis, R. 2008. The Study of Second Language Acquisition (Second edition). Oxford : Oxford University Press.

Faerch, C. 1986. «Rules of Thumb and Other Teacher-formulated Rules in the Foreign Language Classroom », in Kasper, G., ed., Language, Teaching and Communication in the Foreign Language Classroom, Aarhus, Aarhus University Press : 125-143. 
Field, J. 2004. « An insight into listeners' problems : too much bottom-up or too much top-down? ». System, $32: 363-377$.

Gernsbacher, M. A. 1990. Language Comprehension as Structure Building. Hillsdale, N.J. : Erlbaum.

Griggs, P., P. Bange \& R. Carol. 2002. "La dimension cognitive dans l'apprentissage des langues étrangères ». Revue française de linguistique appliquée, $\operatorname{VII}(2 / 2002)$ : 25-38.

Hansen, C. \& C. Jensen. 1994. Evaluating lecture comprehension. In Flowerdew, J. (ed.). Academic Listening. Cambridge : Cambridge University Press, 241-268.

Howard, D. 1985. Cognitive psychology. New York : Macmillan.

Kellerman, E. 1979. "Giving learners a break : native language intuitions as a source of predictions about transferability ». Working Papers on Bilingualism, $15: 37-57$.

Long, D. R. 1989. «Second language listening comprehension : A schematheoretic perspective ». Modern Language Journal, $73: 32-40$.

O’Malley, J. M. \& A. U. Chamot. 1990. Learning Strategies in Second Language Acquisition. Cambridge : Cambridge University Press.

Oxford, R. L. 2011. Teaching and Researching Language Learning Strategies. Harlow : Pearson education.

Paradis, M. 2009. Declarative and Procedural Determinants of Second Languages. Amsterdam : John Benjamins Publishing Company.

Perfetti, C. A. 1985. Reading Ability. Oxford : Oxford University Press.

Ringbom, H. 2002. Levels of transfer from L1 and L2 in L3-acquisition. In J. Ysma et M. Hooghiemstra (eds.). Proceedings of the Second International Conference on Trilingualism. Leeuwaarden : Fryske Akademie, CD-Rom.

Sagnier, C. 2012. "Vers une didactique de la cognition située? Repenser les travaux sur l'apprenant ». Ela. Études de linguistique appliquée, 2012/4(168) : 407-416.

Selinker, L., \& B. Baumgartner-Cohen. 1995. «Multiple Language Acquisition : 'Damn it, why can't I keep these two languages apart?' ». Language, Culture and Curriculum, 8/2:115-121.

Sockett, G. 2001. "Quel fondement théorique pour les stratégies d'apprentissage cognitives? ». Mélanges CRAPEL, $26: 185-193$.

Stanovich, K. E. 1980. «Toward an interactive-compensatory model of individual differences in the development of reading fluency ». Reading Research Quarterly, $16: 32-71$. 